\title{
Supraorbital morphology and social dynamics in human evolution
}

\author{
Ricardo Miguel Godinho $\oplus^{1,2 \star}$, Penny Spikins $\circledast^{3}$ and Paul O'Higgins $\circledast^{1}$
}

\begin{abstract}
Uniquely, with respect to Middle Pleistocene hominins, anatomically modern humans do not possess marked browridges, and have a more vertical forehead with mobile eyebrows that play a key role in social signalling and communication. The presence and variability of browridges in archaic Homo species and their absence in ourselves have led to debate concerning their morphogenesis and function, with two main hypotheses being put forward: that browridge morphology is the result of the spatial relationship between the orbits and the brain case; and that browridge morphology is significantly impacted by biting mechanics. Here, we virtually manipulate the browridge morphology of an archaic hominin (Kabwe 1), showing that it is much larger than the minimum required to fulfil spatial demands and that browridge size has little impact on mechanical performance during biting. As browridge morphology in this fossil is not driven by spatial and mechanical requirements alone, the role of the supraorbital region in social communication is a potentially significant factor. We propose that conversion of the large browridges of our immediate ancestors to a more vertical frontal bone in modern humans allowed highly mobile eyebrows to display subtle affiliative emotions.
\end{abstract}

W hy anatomically modern humans lack a pronounced supraorbital ridge while our Middle Pleistocene ancestors possessed one is an unresolved debate, with the focus on structural and mechanical rather than social signalling roles. The spatial hypothesis considers browridges to be "only a reflection of the spatial relationship between two functionally unrelated cephalic components, the orbit and the brain case"1 (p.281). Additionally, brain and basicranial morphology $y^{2-4}$ and the orientation of the face relative to the cranial vault influence browridge morphology $y^{5}$. Browridges also scale allometrically, with individuals of bigger species growing proportionally bigger ones ${ }^{6,7}$. However, basicranial morphology, facial hafting ${ }^{8}$ and facial size differ little between Kabwe 1 (Homo heidelbergensis, dated from 125-300 kyr $\mathrm{BP}^{9}$ ) and Neanderthals and so do not explain why the comparably large faces of near relatives such as Neanderthals do not manifest equally massive browridges. In contrast, the differences between these archaic members of our genus and modern humans in brow morphology may well relate to gracilization, our reduced facial size and its allometric consequences.

Importantly, the cranial gracilization that humans underwent has also been associated with prosociality ${ }^{10,11}$. Selection for increased sociality and tolerance has been argued to be associated with evolutionary changes in cranial form (that is, the reduction of browridge and upper facial size) via changes in hormonal reactivity that have pleiotropic effects on skeletal form, physiology and behaviour, termed 'self-domestication' ${ }^{10,11}$ (sensu ref. ${ }^{12}$ ). This hypothesis finds support from several studies of non-human mammals (dogs versus wolves, selected versus non-selected foxes, and bonobos versus chimpanzees) that have been able to demonstrate that domestication and increased social tolerance trigger a set of changes that include physiological, morphological and behavioural variables (for a review, see ref. ${ }^{11}$ ).

This association between cranial gracilization, prosociality and self-domestication has also been hypothesized for bonobos who, relative to chimpanzees, present a gracile cranium ${ }^{13}$ with smaller browridges $^{14}$ and prosocial behaviour, and are hypothesized as self-domesticated ${ }^{11,12}$. Thus, this suggests a selective trade-off between expressing dynamic affiliative signals and permanent competitive signals that affects the shape and size of the cranium in general and the browridge in particular. More affiliative-based social relationships in bonobos, with frequent consolation ${ }^{15}$, are associated with both a reduced browridge and greater attention to the eye area in social communication ${ }^{16}$ than in common chimpanzees. Despite this association, it should be noted that bonobos are significantly smaller than chimpanzees ${ }^{13}$ and that, as predicted by the allometric hypothesis ${ }^{6}$, browridges are expected to be proportionally smaller.

For modern humans, gracilization and reduction of the facial skeleton result in significant changes to the supraorbital region, rendering the contour between the orbits and forehead more vertical and smooth. For the frontalis belly of the occipito-frontalis, there are particular consequences. We note that its vector of action changes to be more vertical and this means the eyebrows have the potential to move vertically over a relatively larger area, and to be more readily observed and more mobile (Supplementary Fig. 1).

Alternatively, the mechanical hypothesis explains larger brows in terms of resistance to masticatory loadings. While not necessarily opposed to the spatial hypothesis, it posits that mechanical loadings experienced by the skull during biting and food pre-processing $^{17-21}$ impact decisively on the morphology of browridges ${ }^{21}$. Studies focusing on fossil hominins ${ }^{22}$, extant humans ${ }^{21,23,24}$ and other extant non-human primates ${ }^{25-27}$ support this hypothesis, while it has been challenged by studies of non-human primates that failed to record elevated strains in the browridge during masticatory system loading ${ }^{25-27}$.

In addition to the above, other hypotheses have been proposed to explain large browridges. These include protection from blows to the head ${ }^{28,29}$, protection of the eyes in aquatic environments ${ }^{30}$,

'Department of Archaeology and Hull York Medical School, University of York, York, UK. ${ }^{2}$ Interdisciplinary Center for Archaeology and Evolution of Human Behaviour, Faculdade das Ciências Humanas e Sociais, Universidade do Algarve, Faro, Portugal. ${ }^{3}$ Department of Archaeology, University of York, York, UK. *e-mail: ricardomiguelgodinho@gmail.com 
provision of sunshade ${ }^{31}$ and prevention of hair from obscuring vision ${ }^{32}$, but have not been strongly supported by evidence and so are not widely held as feasible. Another factor that could explain the morphology of the browridge of Kabwe 1 is its massive frontal sinus. However, the sinus appears to have no critical mechanical function during biting ${ }^{33,34}$ and grows and develops secondarily to the browridge ${ }^{35}$.

Thus, after several decades of research, conflicting views still exist with regard to the mechanisms that give rise to large or small browridges and their function. Hypotheses that link the development of modern human browridge morphology to changes in sociality have tended to be set aside in favour of mechanical and spatial ones, aiming to explain large browridges rather than the causes and consequences of small ones.

While there is strong support for a spatial explanation of larger browridges in archaic versus modern humans in that facial reduction reduces the need for large brows to accommodate the orbitofrontal junction, this does not explain why the browridge of Kabwe 1 is much larger than that of Neanderthals despite a generally similar facial size. This may be for mechanical reasons, as noted above, or it may be for other reasons such as social communication.

If it can be shown that the browridge of Kabwe 1 is much larger than spatial requirements demand and has no mechanical function, explanations of its size in terms of social communication become more tenable and the consequences of interactions of small brows in modern humans with sociality, display and social communication become a focus of interest.

One of the reasons that spatial and mechanical explanations of large brows in archaic humans have not been falsified is because of the impossibility of carrying out in vivo experimental manipulations. However, recent advances in virtual functional simulation offer a way forward ${ }^{36-38}$. Through virtual modelling and manipulation of the Kabwe 1 cranium, we show that the browridge is much larger than the minimum size required to accommodate the disjunction between the orbits and frontal bone. Thus, spatial requirements do not fully explain the browridge of this specimen.

Improved craniofacial resistance to masticatory loads, as a consequence of the larger-than-needed browridge, was also assessed using finite-element analysis. This allowed us to virtually manipulate the morphology of the browridge while simulating masticatory system loadings to assess the impact of variations in form on functional performance. Thus, the skull of Kabwe 1 was virtually reconstructed to restore its original morphology ${ }^{39}$ and two additional versions of the model were created in which the form of the browridge was progressively reduced to the minimum required to bridge the gap between the face and neurocranium (simulating the spatial hypothesis $\left.{ }^{1}\right)$. Finite-element models were then created and loaded to simulate biting to assess the impact of different browridge morphologies on the biomechanical performance of the facial skeleton of Kabwe 1. This specimen was used in this study because it presents an extremely well-developed-indeed iconic-browridge.

Our findings show that the browridge of Kabwe 1 is larger than needed to fulfil spatial requirements in accommodating the orbitofrontal junction and that it has no marked role in resisting masticatory loading. As such, sociality and social communication must be considered in relation to both the larger-than-needed browridge of Kabwe 1 and the reduced browridges and more vertical forehead of modern humans.

\section{Results}

The browridge can be much reduced in size, but not eliminated, without creating any significant disjunction between the orbits and the frontal bone (Fig. 1). Thus, while the spatial relationship between the orbits and frontal bone ${ }^{1,2}$ partly explains the large browridge of Kabwe 1, it appears to greatly exceed what would be required to simply bridge the gap (spatial model).
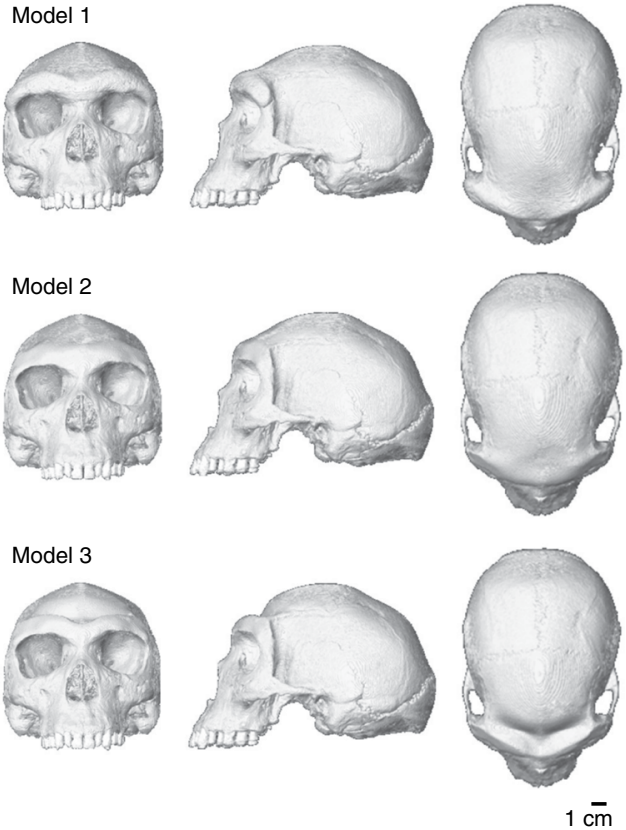

Fig. 1 | Models 1-3. Model 1 represents the original reconstruction of Kabwe 1. Model 2 represents the reconstruction of Kabwe 1 with a reduced browridge. Model 3 represents the reconstruction of Kabwe with a reduced browridge and a post-orbital sulcus.

Furthermore, when models with reduced browridges are compared with the original browridge there are no marked intra-bite differences in strain magnitudes and orientations, whereas interbite comparisons show clear differences in strain magnitudes and orientations (Figs. 2 and 3). Visual examination of strains experienced by the cranium indicates a slight increase in the strain magnitudes experienced by the lateral margins of the ridges and over the frontal bone with decreasing browridge size. This increase in strain magnitudes is most marked over the post-orbital sulcus of the model with the smallest browridge (Fig. 2). It is unknown whether this would be sufficient for biomechanical bone adaptation to occur, as predicted by the mechanostat model ${ }^{40}$. Thus, it is possible that to some extent the growth and development of the browridge may be mechanically driven. However, the increases in strain magnitudes resulting from progressive reduction of the browridge are slight and thus unlikely to fully explain the massive browridge of Kabwe 1.

When considering strains experienced by the face under the same bite, only very small differences were found between models (Fig. 4). The geometric morphometric analysis of changes in size and shape shows that loaded models cluster tightly by bite rather than browridge morphology (Fig. 5). Thus, the vectors of deformation (changes in size and shape) connecting the unloaded and loaded models reflect almost identical modes and magnitudes of deformation in the same bite, irrespective of browridge morphology.

\section{Discussion}

These results demonstrate that the browridge is significantly larger than required to simply bridge the gap between the orbits and the frontal bone. Furthermore, changing the morphology of the browridge does not impact in any substantial way the mode or magnitude of deformation experienced by the face during biting. As such, we falsify spatial ${ }^{1}$ and mechanical ${ }^{17-21}$ hypotheses as complete explanations of the large browridge of this fossil. Rather, the findings suggest that the browridge in Kabwe 1 probably had additional causes.

Relevant in this regard is ref. ${ }^{41}$, which shows that facial bony structures such as the paranasal swellings in Mandrillus sphinx form due to factors that are neither spatial nor mechanical. Rather, 


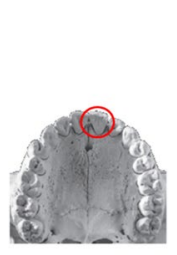

Upper left central incisor

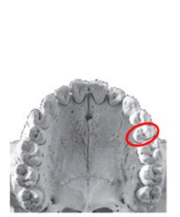

Upper left second premolar

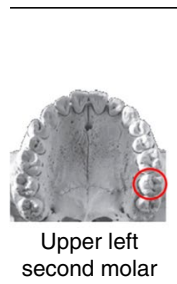

Column
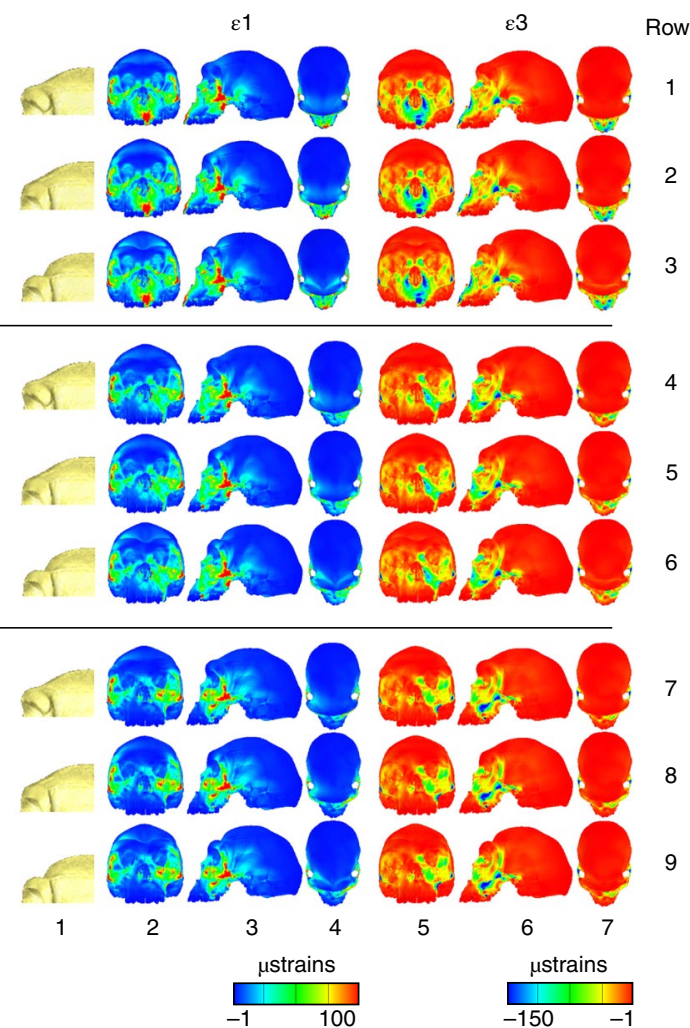

Fig. 2 | Strain contour plots of the biting simulations. Maximum principal strains $(\varepsilon 1)$ are represented in columns 3-5, with minimum principal strains ( $\varepsilon 3$ ) in columns 5-7. Model 1 is represented in rows 1, 4 and 7, with model 2 in rows 2, 5 and 8, and model 3 in rows 3,6 and 9. The different simulated bites are shown to the left.

they reflect social behaviour and structure; these structures underlie the vibrant soft tissue colourings of the muzzle of male mandrills, which bear an important function in social signalling and display ${ }^{42,43}$. Growth and development of the swellings in Mandrillus leucophaeus have been related to androgen production $^{44}$. In humans, the browridge is a sexually dimorphic anatomical trait ${ }^{45}$ that has been identified as relevant in the perception of an individual by others ${ }^{46,47}$ and its growth and development have also been related to androgen production, along with general facial sexual dimorphism ${ }^{48}$. In this regard, we note that the vermiculate bone found over the browridge of Kabwe 1 presents macroscopic similarities to the bone found in the paranasal swellings of Mandrillus species. Although vermiculate bone is less frequent in modern humans than other middle and late Pleistocene hominins ${ }^{28}$, it is more frequent in men than women ${ }^{49}$ and hence its formation is probably related to hormonal factors. It is therefore plausible that the morphology of the browridge of Kabwe 1 might also be related to factors such as sexually dimorphic display and social signalling. Like antlers, they are fixed and have been hypothesized to signal dominance or aggression?.

Facial reduction in Homo sapiens, which has been related to changes in brain and basicranial morphology $y^{2-4}$, as well as food pre-processing and biting mechanics ${ }^{17-21}$, is accompanied by gracilization of the brows, and the development of a more vertical frontal bone. The upper facial morphological changes found in $H$. sapiens position the frontal bone more vertically, inevitably altering the mechanical functioning of the frontalis belly of the occipito-frontalis muscle, causing contraction to raise the supraorbital skin, whereas previously it would have pulled it more posteriorly over the browridge and the low, more horizontal forehead (Supplementary Fig. 1). Having lost a large, low browridge, our

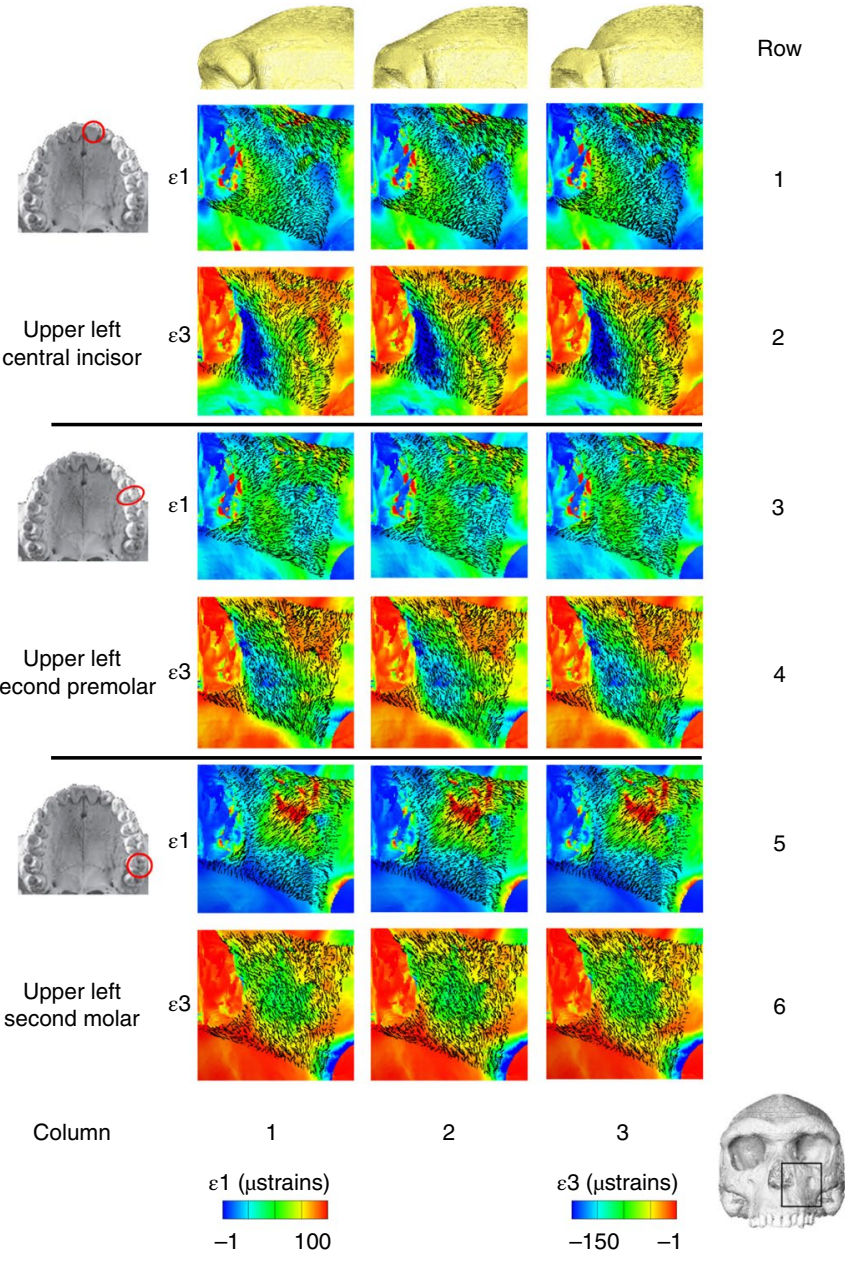

Fig. 3 | Strain contour plots and strain directions. Maximum principal strains ( $\varepsilon$; rows 1, 3 and 5 ) and minimum principal strains ( $\varepsilon 3$; rows 2,4 and 6 ) over the maxilla (see inset frontal view for location) are shown for the different models (left, model 1; middle, model 2; right, model 3) under the different bites simulated. The bottom left inset shows the anatomical region included in vector plots.

ancestors gained the possibility of greater range, subtlety and visibility with regards to movement of the skin overlying the frontal bone, particularly affecting movements of the eyebrow. This suggestion is consistent with ref. ${ }^{50}$, which suggests that the absence of specific movements of the brows in chimpanzees compared with humans may relate to the presence of large browridges (see below). Effectively, these anatomical changes enhance the capacity of the frontalis muscle to move eyebrows over the frontal-a key component of social signalling and non-verbal communication in our highly socially complex species.

Our mobile hairy eyebrows are crucial in subtle signalling behaviours. The eye region is known to develop increasing social significance in a human evolutionary context ${ }^{51,52}$; however, the mobility of eyebrows specifically has received little attention. Mobile eyebrows without the constraints of a pronounced browridge allow subtle affiliative emotions to be expressed (Supplementary Table 3), such as the rapid 'eyebrow flash', lasting around one-sixth of a second, found cross-culturally as a sign of contact readiness and recognition $^{53}$. In contrast, a slow eyebrow raise is a sign of surprise and, in particular, social indignation ${ }^{54}$. The facial expression of sympathyshown by pulling eyebrows up at the middle ${ }^{55}$ - has the advantage of removing need for the direct contact used to express sympathy in chimpanzees ${ }^{56}$. Subtle dynamic movements of eyebrows are also 

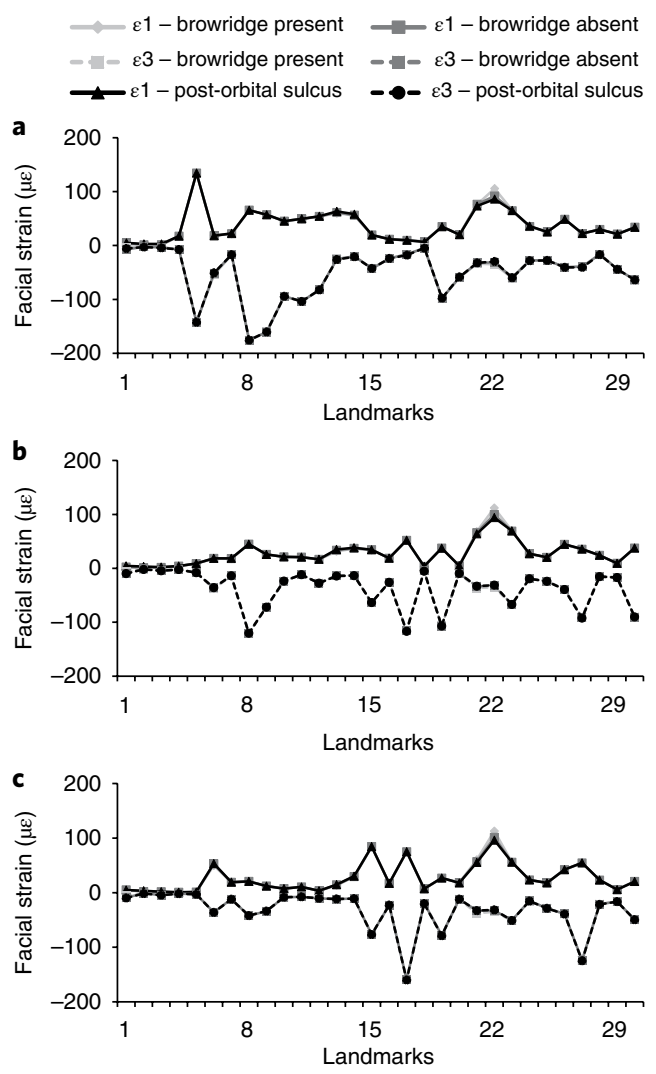

Fig. 4 | Facial strains experienced by the models at $\mathbf{3 0}$ anatomical points. $\mathbf{a}-\mathbf{c}$, Plots of strains for landmarks of the upper left central incisor (a), upper left second premolar (b) and upper left second molar bite (c).

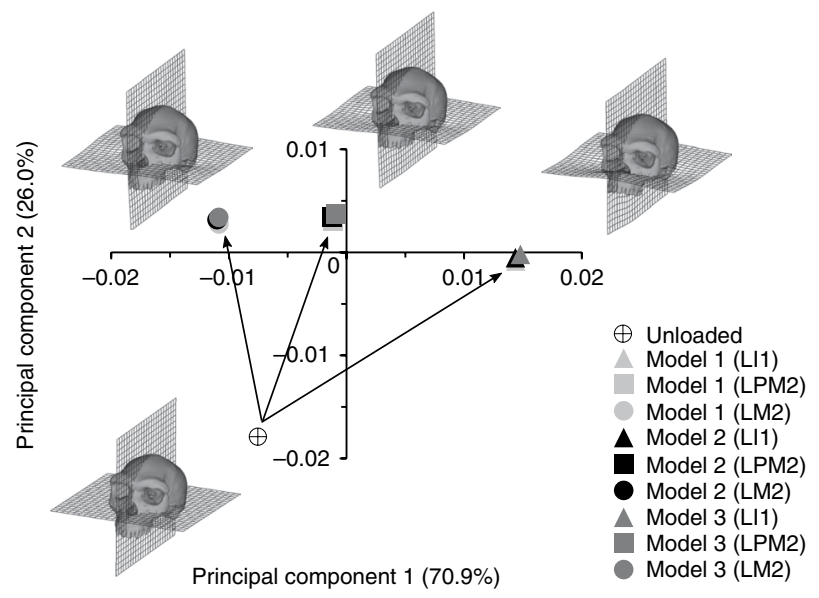

Fig. 5 | Size and shape principal components analysis. Unloaded and loaded models are shown for the three different simulated bites (LII, left central incisor; LM2, left second molar; LPM2, left second premolar).

a key component of identifying trustworthiness ${ }^{57}$ as well as subtle indications of deception. Any constraints on muscle movements in the supraorbital region affect emotional expressions and, in turn, social relationships; for example, individuals who receive a cosmetic procedure (botox) that reduces muscle activity in the forehead (and so affects eyebrow movement) are less able to empathise with and identify other's emotions ${ }^{58}$.

Compared with our own species, our nearest living relatives, chimpanzees, show minimal differences in underlying facial musculature $^{59}$; however, differences in facial morphology, pigmentation and other superficial characteristics impact on the range and sub- tlety of their emotional expressions ${ }^{50,60}$. As in humans, chimpanzees express emotions through the stretching of skin across prominent browridges, but lack the subtleties in eyebrow movement and signalling that modern humans display. This is apparent through the inability of chimpanzees to move the inner and outer brows independently (activated by the medial and lateral parts of the frontalis muscle, respectively) and to present the 'brow lower' action (activated by the corrugator, depressor supercilli and procerus muscles, and significant in identifying sadness and anger in humans) ${ }^{50,60}$. The absence of these movements has been associated with the presence of a large browridge, which precludes marked saliency of these movements and thus of signalling function to conspecifics $^{50}$. Similarly, other non-human primates, such as macaques ${ }^{61}$, gibbons $^{62}$ and orangutans $s^{63}$, are also unable to move their inner and outer brows independently and display brow-lowering (excluding orangutans, which are able to perform brow-lowering). Moreover, human eyebrows overlie a vertically flatter brow and hairless forehead, hence increasing eyebrow visibility and signalling ${ }^{60}$.

The relative selective trade-offs between a pronounced browridge (a permanent social signal) and capacities to dynamically express affiliative prosocial emotions through highly mobile eyebrows are complex. Moreover, competitive and collaborative strategies typically exist together and vary dynamically through time and space $^{64}$. Even in modern hunter-gatherers, more competitive and collaborative individuals tend to spatially locate together ${ }^{65}$. We should thus expect a long period of differing facial forms, reflecting differing social strategies, both within and between groups before the selective advantages of expressing complex prosocial emotions become stable. This pattern seems typical of archaic humans, with substantial variability in the definition of browridges among early modern humans at Jebel Irhoud, for example ${ }^{66}$.

\section{Methods}

The Kabwe 1 cranium reconstruction ${ }^{39}$ was based on a computed tomography scan provided by the Natural History Museum, London (courtesy of R. Kruszynski). After reconstruction, two additional models were created in which the morphology of the browridge was the only anatomical region modified. The models were then directly converted into voxel-based finite-element models and used to simulate three different bites (the left central incisor, left second premolar and left second molar) to assess the biomechanical performance of the facial skeleton during these bites.

Skull reconstruction and model creation. A complete description of the reconstruction of Kabwe 1 is presented in ref. ${ }^{39}$. Briefly, automated, semiautomated and manual segmentation of the cranium was performed using Avizo (version 7.0). Manual segmentation was required to remove sedimentary matrix present in the maxillary and sphenoidal sinuses. When possible, reconstruction of missing parts was performed by mirroring preserved contralateral elements and warping them to the existing structures. When small gaps were present, Geomagic (Studio 2011) was used to fill them using the surface of surrounding structures as the reference for interpolation. Portions of a computed tomography reconstruction of a cadaveric $H$. sapiens skull were used to reconstruct part of the occipital and missing tooth crowns for which there were no preserved antimeres.

Once the reconstruction was complete (model 1), the frontal sinuses were infilled to allow later excavation of this region to produce variant morphologies. Analysis of the impact of infilling the sinus in model 1 showed that the surface strains over the browridge and elsewhere in the cranium did not differ significantly between the models with hollow and filled frontal sinuses ${ }^{34}$. The morphology of the browridge was manipulated using Geomagic by decreasing its size (model 2) and creating a post-orbital sulcus in model 3 (Fig. 1). Voxel-based finite-element models were then generated by direct conversion using bespoke software.

Constraints. Identical constraints were applied to all models using the finiteelement analysis software tool VoxFE ${ }^{67}$. The models were constrained at the temporomandibular joints (laterally, superoinferiorly and anteroposteriorly) and a third constraint was applied at the simulated bite point (superoinferiorly) in each of the biting simulations (left central incisor, left second premolar and left second molar).

Material properties. Following previous sensitivity studies that showed only local effects of differentiating the material properties of teeth and the surrounding bone, these were assigned the same material properties in all the models used 
in this study. Furthermore, sensitivity analyses that assessed the effect of model simplifications in a human cadaveric cranium ${ }^{68}$, a cranium of Macaca fascicularis ${ }^{69}$ and a varanoid lizard mandible ${ }^{70}$ show that infilling of trabecular bone stiffens the skull and so reduces strain magnitudes, but that the distribution of regions of high and low strain as well as global modes (rather than magnitudes) of deformation are not much affected. Allocating teeth the same material properties as bone has the effect of locally reducing strain gradients in the alveolar region, with little effect elsewhere. This is relevant to the present study because trabecular bone is neither well enough preserved nor imaged at sufficient resolution to accurately represent it in a finite-element model, and the dentition was incomplete and required reconstruction. As such, in all models, trabecular bone and teeth were no separately represented and were allocated the same material properties as cortical bone. Based on previous sensitivity analyses, we expect this to have little impact on the mode of deformation of the loaded cranium, but to reduce the degree to which it deforms.

Cortical bone, trabecular bone and the teeth were allocated isotropic properties, with a Young's modulus of $17 \mathrm{Gpa}$ and a Poisson's ratio of 0.3 . The modulus of elasticity was derived from nanoindentation studies of cortical bone in a cadaveric $H$. sapiens skull ${ }^{68}$. The resulting value of $17 \mathrm{Gpa}$ is within the range of values found in previous studies ${ }^{71,72}$

Muscle loads. Loads were applied to the model to represent the actions of six muscles active during biting: the right and left temporalis, right and left masseter, and right and left medial pterygoid. Absence of the mandible precluded direct estimation of the direction of muscle force vectors and estimation using bony proxies of anatomical cross-sectional areas (and so maximum forces) of muscles that attach to the mandible (masseter and medial pterygoid). However, given that three versions of the same model with identical loads and constraints were compared, it matters little that the applied muscle force vectors approximated rather than replicated physiological loadings. Of significantly more importance was the fact that these forces were identical between models and so did not, themselves, produce differences in strains (modes of deformation) between models. As such, the maximum estimated muscle forces estimated from a $H$. sapiens cadaveric head were applied identically to each model $^{68}$ (Supplementary Table 1). The directions of muscle force vectors were estimated by scaling a Homo neanderthalensis mandible (the Tabun 1 specimen) to the Kabwe 1 skull. These directions were applied to all models and simulations. While this mandible is not from the same fossil, it provides a reasonable estimate of muscle vectors. The impact of error in the estimation of the orientation of the muscle vectors was assessed in a sensitivity analysis in which muscle vectors were varied through $5^{\circ}$ anteroposteriorly and mediolaterally. The results showed that regions of high and low strain varied very little in location (Supplementary Fig. 3) while the average magnitude of strains varied from $\sim 2 \%$ in mediolateral manipulation to $\sim 5 \%$ in anteroposterior changes (Supplementary Table 4).

Model solution and analysis. Finite-element models 1-3 were solved using $\mathrm{VoxFE}^{67}$. The resulting deformations of the finite-element models were compared through (1) visual assessment of strain magnitudes and directions of maximum ( $\varepsilon 1)$ and minimum ( $\varepsilon 3)$ principal strains, (2) plotting of $\varepsilon 1$ and $\varepsilon 3$ at 30 nodes (points) located in the facial skeleton that were common to all models (Supplementary Fig. 2) and (3) analysis of changes in the size and shape between loaded and unloaded models of a configuration of 33 landmarks (points) from the whole cranium (Supplementary Fig. 3 and Supplementary Table 2). The size and shape analysis employed geometric morphometrics to compare changes in size and shape between the unloaded and loaded models. This consisted of an initial registration step comprising scaling to unit size followed by translation of landmark configurations to their centroids, with subsequent rotation to minimize the sum of squared distances between each scaled, translated configuration and the mean configuration. This was followed by rescaling of each configuration to its original centroid size and a principal components analysis of the resulting size and shape coordinates ${ }^{73,74}$. This analysis led to a quantitative comparison of global model deformations (changes in size and shape) in terms of the directions (modes) and magnitudes (degree or extent) of deformation arising from loading.

Reporting Summary. Further information on experimental design is available in the Nature Research Reporting Summary linked to this article.

Data availability. The data that support the findings of this study are available from the authors but restrictions apply to the availability of these data, which were used under license from the Natural History Museum (London) for the current study, and so are not publicly available. Data are, however, available from the authors upon reasonable request and with permission from the Centre for Human Evolution Studies at the Natural History Museum.

Received: 7 December 2016; Accepted: 5 March 2018; Published online: 9 April 2018

\section{References}

1. Moss, M. L. \& Young, R. W. A functional approach to craniology. Am. J. Phys. Anthropol. 18, 281-292 (1960).
2. Enlow, D. H. \& Hans, M. G. Essentials of Facial Growth (W. B. Saunders Company, Philadelphia, 1996).

3. Bastir, M. \& Rosas, A. Cranial base topology and basic trends in the facial evolution of Homo. J. Hum. Evol. 91, 26-35 (2016).

4. Bastir, M. et al. Effects of brain and facial size on basicranial form in human and primate evolution. J. Hum. Evol. 58, 424-431 (2010).

5. Shea, B. T. On aspects of skull form in African apes and orangutans, with implications for hominoid evolution. Am. J. Phys. Anthropol. 68, 329-342 (1985)

6. Ravosa, M. J. Browridge development in Cercopithecidae-a test of 2 models Am. J. Phys. Anthropol. 76, 535-555 (1988).

7. Freidline, S. E., Gunz, P., Harvati, K. \& Hublin, J. J. Middle Pleistocene human facial morphology in an evolutionary and developmental context. J. Hum. Evol. 63, 723-740 (2012).

8. Lieberman, D. E., McBratney, B. M. \& Krovitz, G. The evolution and development of cranial form in Homo sapiens. Proc. Natl Acad. Sci. USA 99, 1134-1139 (2002).

9. Stringer, C. The Origin of Our Species (Penguin, London, 2012).

10. Cieri, R. L., Churchill, S. E., Franciscus, R. G., Tan, J. \& Hare, B. Craniofacial feminization, social tolerance, and the origins of behavioral modernity. Curr. Anthropol. 55, 419-443 (2014).

11. Hare, B. Survival of the friendliest: Homo sapiens evolved via selection for prosociality. Annu. Rev. Psychol. 68, 155-186 (2017).

12. Hare, B., Wobber, V. \& Wrangham, R. The self-domestication hypothesis: evolution of bonobo psychology is due to selection against aggression. Anim. Behav. 83, 573-585 (2012)

13. Shea, B. T. The Pygmy Chimpanzee: Evolutionary Biology and Behavior (ed. Susman, R. L.) 89-130 (Springer, New York, 1984).

14. Coolidge, H. J. Pan paniscus. Pigmy chimpanzee from south of the Congo River. Am. J. Phys. Anthropol. 18, 1-59 (1933).

15. Clay, Z. \& de Waal, F. B. M. Development of socio-emotional competence in bonobos. Proc. Natl Acad. Sci. USA 110, 18121-18126 (2013).

16. Kano, F., Hirata, S. \& Call, J. Social attention in the two species of Pan: bonobos make more eye contact than chimpanzees. PLOS ONE 10, e0129684 (2015)

17. Zink, K. D. \& Lieberman, D. E. Impact of meat and Lower Palaeolithic food processing techniques on chewing in humans. Nature 531, 500-503 (2016).

18. Zink, K. D., Lieberman, D. E. \& Lucas, P. W. Food material properties and early hominin processing techniques. J. Hum. Evol. 77, 155-166 (2014).

19. Carlson, D. S. Temporal variation in prehistoric Nubian crania. Am. J. Phys. Anthropol. 45, 467-484 (1976).

20. Carlson, D. S. \& Van Gerven, D. P. Masticatory function and post-pleistocene evolution in Nubia. Am. J. Phys. Anthropol. 46, 495-506 (1977).

21. Russell, M. D. The supraorbital torus-a most remarkable peculiarity. Curr. Anthropol. 26, 337-360 (1985).

22. Oyen, O. J., Rice, R. W. \& Cannon, M. S. Browridge structure and function in extant primates and Neanderthals. Am. J. Phys. Anthropol. 51, 83-96 (1979).

23. Endo, B. Analysis of stresses around the orbit due to masseter and temporalis muscles respectively. J. Anthropol. Soc. Nippon 78, 251-266 (1970).

24. Hilloowala, R. A. \& Trent, R. B. Supraorbital ridge and masticatory apparatus II: humans (Eskimos). Hum. Evol. 3, 351-356 (1988).

25. Ravosa, M. J., Noble, V. E., Hylander, W. L., Johnson, K. R. \& Kowalski, E. M. Masticatory stress, orbital orientation and the evolution of the primate postorbital bar. J. Human. Evol. 38, 667-693 (2000).

26. Hylander, W. L., Picq, P. G. \& Johnson, K. R. Masticatory-stress hypotheses and the supraorbital region of primates. Am. J. Phys. Anthropol. 86, 1-36 (1991).

27. Kupczik, K. et al. Assessing mechanical function of the zygomatic region in macaques: validation and sensitivity testing of finite element models. J. Anat. 210, 41-53 (2007)

28. Tappen, N. C. The vermiculate surface pattern of brow ridges in Neandertal and modern crania. Am. J. Phys. Anthropol. 49, 1-10 (1978).

29. Tappen, N. C. Structure of bone in the skulls of Neanderthal fossils. Am. J. Phys. Anthropol. 38, 93-97 (1973).

30. Verhaegen, M. The aquatic ape evolves: common misconceptions and unproven assumptions about the so-called aquatic ape hypothesis. Hum. Evol. 28, 237-266 (2013).

31. Clark, G. A. \& Willermet, C. M. Conceptual Issues in Modern Human Origins Research (Evolutionary Foundations for Human Behavior) (Walter de Gruyter, New York, 1997).

32. Krantz, G. S. Cranial hair and brow ridges. Mankind 9, 109-111 (1973).

33. Witzel, U. in Continuity and Discontinuity in the Peopling of Europe: One Hundred Fifty Years of Neanderthal Study (eds Condemi, S. \& Weniger, G.-C.) 203-211 (Springer, Dordrecht, 2011).

34. Godinho, R. M. \& O'Higgins, P. The biomechanical significance of the frontal sinus in Kabwe 1 (Homo heidelbergensis). J. Hum. Evol. 114, 141-153 (2018).

35. Lieberman, D. in Development, Growth and Evolution-Implications for the Study of the Hominid Skeleton Vol. 20 (eds O'Higgins, P. \& Cohn, M.) 85-122 (Academic Press, London, 2000). 
36. Strait, D. S. et al. Masticatory biomechanics and its relevance to early hominid phylogeny: an examination of palatal thickness using finite-element analysis. J. Hum. Evol. 52, 585-599 (2007).

37. O'Higgins, P. et al. Virtual functional morphology: novel approaches to the study of craniofacial form and function. Evol. Biol. 39, 521-535 (2012).

38. O'Higgins, P. \& Milne, N. Applying geometric morphometrics to compare changes in size and shape arising from finite elements analyses. Hystrix Ital. J. Mammal. 24, 126-132 (2013).

39. Godinho, R. M. \& O’Higgins, P. in Human Remains-Another Dimension: the Application of 3D Imaging in Funerary Context (eds Thompson, T. \& Errickson, D.) 135-147 (Elsevier, London, 2017).

40. Frost, H. M. Bone's mechanostat: a 2003 update. Anat. Rec. A Discov. Mol. Cell. Evol. Biol. 275A, 1081-1101 (2003).

41. Hylander, W. \& Johnson, K. R. in Reconstructing Behavior in the Primate Fossil Record (eds Plavcan, J. M., Kay, R. F., Jungers, W. L. \& Schaik, C. P.) 43-72 (Kluwer Academic/Plenum Publishers, New York, 2002).

42. Setchell, J. M. \& Dixson, A. F. Changes in the secondary sexual adornments of male mandrills (Mandrillus sphinx) are associated with gain and loss of alpha status. Horm. Behav. 39, 177-184 (2001).

43. Wickings, E. J. \& Dixson, A. F. Testicular function, secondary sexual development, and social status in male mandrills (Mandrillus sphinx). Physiol. Behav. 52, 909-916 (1992).

44. Elton, S. \& Morgan, B. Muzzle size, paranasal swelling size and body mass in Mandrillus leucophaeus. Primates 47, 151-157 (2006).

45. Buikstra, J. \& Ubelaker, D. Standards for Data Collection from Human Skeletal Remains: Proceedings of a Seminar at the Field Museum of Natural History (Arkansas Archeological Survey, Fayetteville, 1994).

46. Todorov, A., Baron, S. G. \& Oosterhof, N. N. Evaluating face trustworthiness: a model based approach. Soc. Cogn. Affect. Neurosci. 3, 119-127 (2008).

47. Xu, F. et al. Similarities and differences in Chinese and Caucasian adults' use of facial cues for trustworthiness judgments. PLoS ONE 7, e34859 (2012).

48. Thornhill, R. \& MØller, A. P. Developmental stability, disease and medicine. Biol. Rev. 72, 497-548 (1997).

49. Amorim, A., Umbelino, C. \& Matos, V. in V Jornadas Portuguesas de Paleopatologia: a Saúde e a Doença no Passado (eds Lopes, C. et al.) 15 (Centro de Investigação em Antropologia e Saúde, Coimbra, 2016).

50. Parr, L. A., Waller, B. M., Vick, S. J. \& Bard, K. A. Classifying chimpanzee facial expressions using muscle action. Emotion 7, 172-181 (2007).

51. Tomasello, M., Hare, B., Lehmann, H. \& Call, J. Reliance on head versus eyes in the gaze following of great apes and human infants: the cooperative eye hypothesis. J. Hum. Evol. 52, 314-320 (2007).

52. Kret, M. E. Emotional expressions beyond facial muscle actions. A call for studying autonomic signals and their impact on social perception. Front. Psychol. 6, 711 (2015).

53. Grammer, K., Schiefenhovel, W., Schleidt, M., Lorenz, B. \& Eibl-Eibesfeldt, I. Patterns on the face-the eyebrow flash in crosscultural comparison. Ethology 77, 279-299 (1988).

54. Eibl-Eibesfeldt, I. Human Ethology (Aldine de Gruyter, New York, 1989).

55. Bavelas, J. B., Black, A., Lemery, C. R. \& Mullett, J. "I show how you feel”: motor mimicry as a communicative act. J. Pers. Soc. Psychol. 50, 322-329 (1986).

56. Romero, T., Castellanos, M. A. \& de Waal, F. B. M. Consolation as possible expression of sympathetic concern among chimpanzees. Proc. Natl Acad. Sci. USA 107, 12110-12115 (2010).

57. Hehman, E., Flake, J. K. \& Freeman, J. B. Static and dynamic facial cues differentially affect the consistency of social evaluations. Pers. Soc. Psychol. Bull. 41, 1123-1134 (2015).

58. Neal, D. T. \& Chartrand, T. L. Embodied emotion perception. Soc. Psychol. Pers. Sci. 2, 673-678 (2011).

59. Burrows, A. M., Waller, B. M., Parr, L. A. \& Bonar, C. J. Muscles of facial expression in the chimpanzee (Pan troglodytes): descriptive, comparative and phylogenetic contexts. J. Anat. 208, 153-167 (2006).

60. Vick, S.-J., Waller, B. M., Parr, L. A., Smith Pasqualini, M. C. \& Bard, K. A. A cross-species comparison of facial morphology and movement in humans and chimpanzees using the facial action coding system (FACS). J. Nonverbal Behav. 31, 1-20 (2007).
61. Parr, L. A., Waller, B. M., Burrows, A. M., Gothard, K. M. \& Vick, S. J. Brief communication: MaqFACS: a muscle-based facial movement coding system for the rhesus macaque. Am. J. Phys. Anthropol. 143, 625-630 (2010).

62. Waller, B. M., Lembeck, M., Kuchenbuch, P., Burrows, A. M. \& Liebal, K. GibbonFACS: a muscle-based facial movement coding system for hylobatids. Int. J. Primatol. 33, 809-821 (2012).

63. Caeiro, C. C., Waller, B. M., Zimmermann, E., Burrows, A. M. \& Davila-Ross, M. OrangFACS: a muscle-based facial movement coding system for orangutans (Pongo spp.). Int. J. Primatol. 34, 115-129 (2013).

64. Manapat, M. L., Nowak, M. A. \& Rand, D. G. Information, irrationality, and the evolution of trust. J. Econ. Behav. Organ. 90, S57-S75 (2013).

65. Apicella, C. L., Marlowe, F. W., Fowler, J. H. \& Christakis, N. A. Social networks and cooperation in hunter-gatherers. Nature 481, 497-501 (2012).

66. Hublin, J.-J. et al. New fossils from Jebel Irhoud, Morocco and the pan-African origin of Homo sapiens. Nature 546, 289-292 (2017).

67. Fagan, M. J. et al. Voxel-based finite element analysis-working directly with microCT scan data. J. Morphol. 268, 1071 (2007).

68. Toro-Ibacache, V., Fitton, L. C., Fagan, M. J. \& O'Higgins, P. Validity and sensitivity of a human cranial finite element model: implications for comparative studies of biting performance. J. Anat. 228, 70-84 (2016).

69. Fitton, L. C., Prôa, M., Rowland, C., Toro-Ibacache, V. \& O’Higgins, P. The impact of simplifications on the performance of a finite element model of a Macaca fascicularis cranium. Anat. Rec. 298, 107-121 (2015).

70. Parr, W. C. H. et al. Toward integration of geometric morphometrics and computational biomechanics: new methods for $3 \mathrm{D}$ virtual reconstruction and quantitative analysis of finite element models. J. Theor. Biol. 301, 1-14 (2012).

71. Dechow, P. C., Nail, G. A., Schwartz-Dabney, C. L. \& Ashman, R. B. Elastic properties of human supraorbital and mandibular bone. Am. J. Phys. Anthropol. 90, 291-306 (1993).

72. Schwartz-Dabney, C. L. \& Dechow, P. C. Variations in cortical material properties throughout the human dentate mandible. Am. J. Phys. Anthropol. 120, 252-277 (2003)

73. O'Higgins, P. The study of morphological variation in the hominid fossil record: biology, landmarks and geometry. J. Anat. 197, 103-120 (2000).

74. Zelditch, M. L., Swiderski, D. L., Sheets, H. D. \& Fink, W. L. Geometric Morphometrics for Biologists: A Primer (Elsevier, New York, 2004).

\section{Acknowledgements}

R.M.G. is funded by the Portuguese Foundation for Science and Technology (PhD funding reference: SFRH/BD/76375/2011). We are grateful to L. C. Fitton and S. Cobb at Hull York Medical School, C. Stringer at the Natural History Museum and B. Waller at the University of Portsmouth for discussion about this work. We thank R. Kruszynski at the Natural History Museum for facilitating access to the computed tomography scans and the original fossil of Kabwe 1. We also thank W. Sellers at the University of Manchester for access to software (Geomagic) in his laboratory. We are also grateful to the reviewers for helpful comments and suggestions.

\section{Author contributions}

R.M.G., P.S. and P.O. designed the experiment. R.M.G. performed the simulations. R.M.G., P.S. and P.O. wrote the manuscript.

\section{Competing interests}

The authors declare no competing interests.

\section{Additional information}

Supplementary information is available for this paper at https://doi.org/10.1038/ 441559-018-0528-0.

Reprints and permissions information is available at www.nature.com/reprints. Correspondence and requests for materials should be addressed to R.M.G.

Publisher's note: Springer Nature remains neutral with regard to jurisdictional claims in published maps and institutional affiliations. 


\section{natureresearch}

Corresponding author(s): Ricardo Miguel Godinho

\section{Life Sciences Reporting Summary}

Initial submission $\square$ Revised version \ Final submission

Nature Research wishes to improve the reproducibility of the work that we publish. This form is intended for publication with all accepted life science papers and provides structure for consistency and transparency in reporting. Every life science submission will use this form; some list items might not apply to an individual manuscript, but all fields must be completed for clarity.

For further information on the points included in this form, see Reporting Life Sciences Research. For further information on Nature Research policies, including our data availability policy, see Authors \& Referees and the Editorial Policy Checklist.

\section{- Experimental design}

1. Sample size

Describe how sample size was determined.

Does not apply.

2. Data exclusions

Describe any data exclusions.

No data excluded.

3. Replication

Describe whether the experimental findings were reliably reproduced.

\section{Randomization}

Describe how samples/organisms/participants were allocated into experimental groups.

\section{Blinding}

Describe whether the investigators were blinded to group allocation during data collection and/or analysis.

Does not apply.

Models were run more than once and results consistent between runs.

Does not apply.

Note: all studies involving animals and/or human research participants must disclose whether blinding and randomization were used.

\section{Statistical parameters}

For all figures and tables that use statistical methods, confirm that the following items are present in relevant figure legends (or in the Methods section if additional space is needed).

n/a $\mid$ Confirmed

Х $\square$ The exact sample size ( $n$ ) for each experimental group/condition, given as a discrete number and unit of measurement (animals, litters, cultures, etc.) $\bigotimes \square \begin{aligned} & \text { A description of how samples were collected, noting whether measurements were taken from distinct samples or whether the same } \\ & \text { sample was measured repeatedly }\end{aligned}$

Х $\square$ A statement indicating how many times each experiment was replicated

Х The statistical test(s) used and whether they are one- or two-sided (note: only common tests should be described solely by name; more complex techniques should be described in the Methods section)

Х $\square$ A description of any assumptions or corrections, such as an adjustment for multiple comparisons

Х $\square$ The test results (e.g. $P$ values) given as exact values whenever possible and with confidence intervals noted

Х $\square$ A clear description of statistics including central tendency (e.g. median, mean) and variation (e.g. standard deviation, interquartile range) $\bigotimes \square$ Clearly defined error bars 
Policy information about availability of computer code

\section{Software}

Describe the software used to analyze the data in this study.

VoxFE was used to perform all pre and post-processing of the finite element analysis.

For manuscripts utilizing custom algorithms or software that are central to the paper but not yet described in the published literature, software must be made available to editors and reviewers upon request. We strongly encourage code deposition in a community repository (e.g. GitHub). Nature Methods guidance for providing algorithms and software for publication provides further information on this topic.

\section{- Materials and reagents}

Policy information about availability of materials

\section{Materials availability}

Indicate whether there are restrictions on availability of unique materials or if these materials are only available for distribution by a for-profit company.

\section{Antibodies}

Describe the antibodies used and how they were validated for use in the system under study (i.e. assay and species).

10. Eukaryotic cell lines

a. State the source of each eukaryotic cell line used.

b. Describe the method of cell line authentication used.

c. Report whether the cell lines were tested for mycoplasma contamination.

d. If any of the cell lines used are listed in the database of commonly misidentified cell lines maintained by ICLAC, provide a scientific rationale for their use.
No unique materials were used.

No antibodies were used.

No eukaryotic line cells were used.

No eukaryotic line cells were used.

No eukaryotic line cells were used.

No commonly misidentified cell lines were used.

\section{- Animals and human research participants}

Policy information about studies involving animals; when reporting animal research, follow the ARRIVE guidelines

\section{Description of research animals}

Provide details on animals and/or animal-derived materials used in the study.
No animals were used.

Policy information about studies involving human research participants

\section{Description of human research participants}

Describe the covariate-relevant population characteristics of the human research participants.
The study did not include human research participants. 